PROCEEDINGS OF THE

AMERICAN MATHEMATICAL SOCIETY

Volume 129, Number 7, Pages 1891-1898

S 0002-9939(01)05787-2

Article electronically published on January 23, 2001

\title{
SOME PROPERTIES OF FACTORIZABLE HOPF ALGEBRAS
}

\author{
H.-J. SCHNEIDER
}

(Communicated by Ken Goodearl)

\begin{abstract}
A direct proof without modular category theory is given of a recent theorem of Etingof and Gelaki (1998) on the dimensions of irreducible representations. Factorizable Hopf algebras are characterized in terms of their Drinfeld double, and their character rings and the group-like elements of their duals are described.
\end{abstract}

\section{INTRODUCTION}

Etingof and Gelaki [EG] recently obtained a fundamental result in the representation theory of the Drinfeld double of a semisimple Hopf algebra $H$ over an algebraically closed field of characteristic 0 . Using the theory of modular ribbon categories, they showed that the dimension of any simple $D(H)$-module divides the dimension of $H$.

In this note, a direct proof of their result is given. More generally let $(A, R)$ be a quasitriangular and semisimple Hopf algebra. Assume that $(A, R)$ is factorizable in the sense of [RS] (see section 2). Then by Theorem 3.2, the square of the dimension of any simple $A$-module divides the dimension of $A$. The theorem of Etingof and Gelaki is an immediate corollary by taking $A=D(H)$ with the usual $R$-matrix of the double. The main ingredients of the short proof of Theorem 3.2 are the class equation of Kac and Zhu (see $\mathrm{Q}$ ] for an elementary proof), and Drinfeld's central element construction in [D. Drinfeld's construction is reviewed in section 2 Here it is essential to note (see Theorem[2.1) that Drinfeld's proof of [D] 3.3] shows more than what is actually stated in D, 3.3].

Moreover this paper contains two new results about factorizable Hopf algebras over an arbitrary field. Let $(A, R)$ be a factorizable Hopf algebra. It is not difficult to see that Drinfeld's construction defines an algebra isomorphism between a character ring $C(A)$ and the center $Z(A)$ of $A$. By Theorem 2.3, this isomorphism induces a group isomorphism between the group-like elements of the linear dual $A^{*}$ and the central group-like elements of $A$. This last result answers a question of Sonia Natale. When $A$ is the double of a finite-dimensional Hopf algebra $H$, the group isomorphism in 2.3 was already obtained by Radford [R93, Propositions 9 and 10].

Received by the editors May 20, 1999 and, in revised form, October 22, 1999.

1991 Mathematics Subject Classification. Primary 16W30; Secondary 16G10.

Key words and phrases. Factorizable Hopf algebras, irreducible representations, Drinfeld double. 
In section 4, a new characterization of factorizable Hopf algebras is given. Let $(A, R)$ be a quasitriangular and finite-dimensional Hopf algebra. If $(A, R)$ is factorizable, then it is stated in [RS] (without an explicit proof) that the double $D(A)$ is isomorphic to a 2-cocycle twist of the tensor product Hopf algebra $A \otimes A$. Tsang and Zhu recently rediscovered this result in the case when $A$ is the double of another Hopf algebra $H$. Their proof uses the special structure of the double $D(H)$. In Theorem 4.3 a stronger result is obtained in a more conceptual way: $(A, R)$ is factorizable if and only if the double $D(A)$ is isomorphic (in a specific way) to a 2-cocycle twist of the tensor product $A \otimes A$. The proof of 4.3 follows from the theorem on Hopf modules.

This note originated on the occasion of some lectures I gave at the University of Córdoba, Argentina, in the fall of 1998. I would like to thank Nicolás Andruskiewitsch, the FOMEC and FaMAF for the invitation.

\section{Central and group-like elements}

In this paper, algebras and coalgebras are defined over the ground-field $k$; comultiplication and counit of a coalgebra and the antipode of a Hopf algebra will be denoted by $\Delta, \epsilon$ and $S$. If $A$ is an algebra, the dual vector space $A^{*}$ is an $(A, A)$-bimodule with $(a f)(b)=f(b a)$ and $(f a)(b)=f(a b)$ for all $a, b \in A$.

Let $A$ be a Hopf algebra and $R$ an invertible element in $A \otimes A$. Elements $t$ in $A \otimes A$ will be written symbolically as $t=t^{1} \otimes t^{2}$. Following Drinfeld, the pair $(A, R)$ is called quasitriangular if:

(1) $x_{2} R^{1} \otimes x_{1} R^{2}=R^{1} x_{1} \otimes R^{2} x_{2}$, for all $x \in A$.

(2) $\Delta\left(R^{1}\right) \otimes R^{2}=R^{1} \otimes r^{1} \otimes R^{2} r^{2}$.

(3) $R^{1} \otimes \Delta\left(R^{2}\right)=R^{1} r^{1} \otimes r^{2} \otimes R^{2}$.

Here $R=r$ and the symbolic notations $R=R^{1} \otimes R^{2}=r^{1} \otimes r^{2}$ (to indicate two different summation indices) and $\Delta(x)=x_{1} \otimes x_{2}$ are used. Define $R_{21}=R^{2} \otimes R^{1}=$ $\tau(R)$ where $\tau: R \otimes R \rightarrow R \otimes R$ is the usual twist map, and

$$
b:=R_{21} R=r^{2} R^{1} \otimes r^{1} R^{2} .
$$

Note that $b \Delta(x)=\Delta(x) b$ for all $x \in A$ since $R$ and $R_{21}^{-1}$ both satisfy (1).

The quasitriangular Hopf algebra $(A, R)$ is called factorizable [RS] if the map

$$
\Phi_{R}=\Phi: A^{*} \rightarrow A, \quad \Phi(f):=b^{1} f\left(b^{2}\right) \text { for all } f \in A^{*},
$$

is an isomorphism of vector spaces; $A^{*}$ is the $k$-linear dual of $A$. Important examples of factorizable Hopf algebras are the Drinfeld doubles of arbitrary finite-dimensional Hopf algebras [RS, 2.10] (see 2.4 1) below).

In [D], Drinfeld introduced a basic construction of central elements of a quasitriangular Hopf algebra. As in [D] let

$$
C(A):=\left\{f \in A^{*} \mid \text { for all } x, y \in A: f(x y)=f\left(y S^{2}(x)\right)\right\} .
$$

Let $Z(A)$ denote the center of $A$. The next crucial result is due to Drinfeld [D] 1.2 and 3.3]. However the formulation below in (b) is more general; D, 3.3] just says that the map $\Phi$ defines by restriction an algebra homomorphism on $C(A)$. For completeness the short proof will be repeated (in a different notation).

Theorem 2.1 (Drinfeld $[\mathrm{D}$ ). Let $(A, R)$ be a quasitriangular Hopf algebra and $\Phi=\Phi_{R}: A^{*} \rightarrow A$. Then for all $g \in C(A)$ and $f \in A^{*}$,

(a) $\Phi(g) \in Z(A)$, and

(b) $\Phi(f g)=\Phi(f) \Phi(g)$. 
Proof. (a) For all $g \in C(A)$ and $x \in A$,

$$
\begin{aligned}
x \Phi(g) & =x_{1} b^{1} g\left(S^{-1}\left(x_{3}\right) x_{2} b^{2}\right) \\
& =b^{1} x_{1} g\left(b^{2} x_{2} S\left(x_{3}\right)\right) \quad \text { since } g \in C(A), \text { and } \Delta(x) b=b \Delta(x) \\
& =\Phi(g) x .
\end{aligned}
$$

(b) For all $f \in A^{*}$ and $g \in C(A)$,

$$
\begin{aligned}
\Phi(f g) & =R^{2} r^{1}(f g)\left(R^{1} r^{2}\right) \\
& =R^{2} r^{1} f\left(R_{1}^{1} r_{1}^{2}\right) g\left(R_{2}^{1} r_{2}^{2}\right) \\
& =R^{2} r^{2} s^{1} t^{1} f\left(R^{1} t^{2}\right) g\left(r^{1} s^{2}\right) \quad \text { by }(2) \text { and }(3) \text { with } R=r=s=t \\
& =R^{2} r^{2} s^{1} g\left(r^{1} s^{2}\right) t^{1} f\left(R^{1} t^{2}\right) \\
& =R^{2} \Phi(g) t^{1} f\left(R^{1} t^{2}\right) \\
& =\Phi(f) \Phi(g) \quad \text { since } \Phi(g) \text { is central by (a). }
\end{aligned}
$$

since $\Phi(g)$ is central by (a).

Lemma 2.2. Let $A$ be a finite-dimensional unimodular Hopf algebra. Then $\operatorname{dim}(C(A))=\operatorname{dim}(Z(A))$.

Proof. Let $\lambda \in A^{*}$ be a non-zero left integral. Then $A \rightarrow A^{*}, a \mapsto \lambda a$, is bijective. Since $A$ is unimodular, for all $a, b \in A, \lambda(a b)=\lambda\left(b S^{2}(a)\right)$ OS 3.2, 2)a)]. Hence for all $a, x, y \in A,(\lambda a)\left(y S^{2}(x)\right)=\lambda\left(a y S^{2}(x)\right)=\lambda(x a y)$. Thus $\lambda a \in C(A)$ if and only if for all $x \in A, \lambda(x a)=\lambda(a x)$, that is $a \in Z(A)$.

If $C$ is a coalgebra, then $G(C)=\{c \in C \mid \Delta(c)=c \otimes c, \epsilon(c)=1\}$ will denote the set of all group-like elements of $C$.

Theorem 2.3. Let $(A, R)$ be a factorizable Hopf algebra. Then $\Phi_{R}: A^{*} \rightarrow A$ induces by restriction

(a) an algebra isomorphism $C(A) \rightarrow Z(A)$, and

(b) a group isomorphism $G\left(A^{*}\right) \rightarrow G(A) \cap Z(A)$.

Proof. (a) By [R94, Proposition 3], $A$ is unimodular (for another proof see 4.4 below). By Theorem 2.1, $\Phi_{R}$ defines an injective algebra map $C(A) \rightarrow Z(A)$ which is bijective by Lemma 2.2 .

(b) Let $f \in G\left(A^{*}\right)=\operatorname{Alg}(A, k)$. Then $f \in C(A)$ since $f S^{2}=f$, fS being the inverse of $f$ in the group $G\left(A^{*}\right)$. Hence $\Phi(f) \in Z(A)$ by (a), and

$$
\Phi(f)=R^{2} r^{1} f\left(R^{1} r^{2}\right)=R^{2} f\left(R^{1}\right) r^{1} f\left(r^{2}\right)=u v,
$$

where $u:=R^{2} f\left(R^{1}\right), v:=r^{1} f\left(r^{2}\right)$. It follows easily from (3) and (2) that $u$ and $v$ are group-like elements of $A$. Thus $\Phi$ defines an injective group homomorphism from $G\left(A^{*}\right)$ to $G(A) \cap Z(A)$.

To prove surjectivity of this map take $g \in G(A) \cap Z(A)$. By Lemma $2.2 \lambda g^{-1}$ is in $C(A)$ since $g^{-1}$ is central. Then for all $p \in A^{*}, p\left(\lambda g^{-1}\right)=p(g) \lambda g^{-1}$ in $A^{*}$, since for all $x \in A$,

$$
\left(p\left(\lambda g^{-1}\right)\right)(x)=p\left(x_{1}\right) \lambda\left(g^{-1} x_{2}\right)=((p g) \lambda)\left(g^{-1} x\right)=p(g)\left(\lambda g^{-1}\right)(x),
$$

since $\lambda$ is a left integral. Hence it follows from Theorem[2.1(b) that for all $p \in A^{*}$,

$$
\Phi(p) \Phi\left(\lambda g^{-1}\right)=\Phi\left(p\left(\lambda g^{-1}\right)\right)=p(g) \Phi\left(\lambda g^{-1}\right) .
$$


Therefore the left $A$-module generated by $\Phi\left(\lambda g^{-1}\right)$ is one-dimensional (since $\Phi$ : $A^{*} \rightarrow A$ is bijective). Hence there is an algebra homomorphism $\chi: A \rightarrow k$ with $p(g)=\chi(\Phi(p))$ for all $p \in A^{*}$.

It remains to show that $\Phi(\chi)=g$ or equivalently $\chi(\Phi(p))=p(\Phi(\chi))$ for all $p \in A^{*}$. Define $u:=R^{1} \chi\left(R^{2}\right), v:=r^{2} \chi\left(r^{1}\right)$. For all $p \in A^{*}$,

$$
\begin{gathered}
\chi(\Phi(p))=\chi\left(R^{2}\right) \chi\left(r^{1}\right) p\left(R^{1} r^{2}\right)=p(u v), \text { and } \\
p(\Phi(\chi))=p\left(R^{2} r^{1} \chi\left(R^{1}\right) \chi\left(r^{2}\right)\right)=p(v u) .
\end{gathered}
$$

Thus the claim follows from the equality $u v=v u$. Since $\Phi$ defines an algebra homomorphism $C(A) \rightarrow Z(A), \Phi(\chi)=S(\Phi(\chi S))$; hence

$$
u v=\Phi(\chi)=S\left(r^{1}\right) \chi\left(S\left(r^{2}\right)\right) S\left(R^{2}\right) \chi\left(S\left(R^{1}\right)\right)=v u,
$$

since $R=S\left(R^{1}\right) \otimes S\left(R^{2}\right)$ by [K, VIII.2.4].

Remark 2.4. 1) Let $H$ be a finite-dimensional Hopf algebra and $A=D(H)=$ $H^{* c o p} \otimes H$ the Drinfeld double of $H$. Elements $p \otimes h, p \in H^{*}, h \in H$ of $D(H)$ will be simply denoted by $p h$. Then $H^{* c o p}$ and $H$ are sub Hopf algebras of $D(H)$ and comultiplication and multiplication in $D(H)$ are given by

$$
\Delta(p h)=p_{2} h_{1} \otimes p_{1} h_{2}, \text { and } h p=p\left(S^{-1}\left(h_{3}\right)-h_{1}\right) h_{2},
$$

for all $h \in H, p \in H^{*}[\underline{\mathrm{K}}$, IX.4.1].

Let $\left(e_{i}\right)$ and $\left(f_{i}\right)$ be dual bases in $H$ and $H^{*}$. Then $D(H)$ is quasitriangular with $R$-matrix $R=\sum_{i} e_{i} \otimes f_{i}\left[\underline{\mathrm{K}}\right.$, IX.4.2]. Thus $b=\sum_{i, j} f_{j} e_{i} \otimes e_{j} f_{i}$ and $\Phi(F)=$ $\sum_{i, j} f_{j} e_{i} F\left(e_{j} f_{i}\right)$ for all $F \in D(H)^{*}$. Since the elements $f_{j} e_{i}, 1 \leq i, j \leq n$, and $S\left(f_{i}\right) S\left(e_{j}\right), 1 \leq i, j \leq n$, are bases of $D(H), \Phi S$ is injective. Hence $(D(H), R)$ is factorizable (cf. [RS, Theorem 2.10], [R94, p. 226]).

2) The group isomorphism in Theorem 2.3 can be described explicitly in case $A=D(H)$ with $R$-matrix as in 1). For any $\chi \in G\left(A^{*}\right)=\operatorname{Alg}(A, k), \Phi(\chi)=u v$ with $u=\sum_{i} f_{i} \chi\left(e_{i}\right)$ and $v=\sum_{i} e_{i} \chi\left(f_{i}\right)$. Thus $u \in G\left(A^{*}\right)$ is the restriction of $\chi$ on $H^{*}$, and the group-like element $v \in G(H)$ is defined by $p(v)=\chi(p)$ for all $p \in H^{*}$. Therefore the isomorphism $G\left(D(H)^{*}\right) \rightarrow G(D(H)) \cap Z(D(H))$ of Theorem 2.3 is the isomorphism constructed by Radford [R93, Propositions 9 and 10].

\section{A Direct PRoOf of a theorem of Etingof-Gelaki}

Throughout this section the field $k$ is algebraically closed of characteristic 0 . The Theorem of Etingof-Gelaki [EG, 1.4] will follow from Drinfeld's central element construction in section 2 and the following result by Kac-Zhu (the class equation, see $\mathrm{L}]$ for a more elementary proof).

Theorem 3.1 (Kac-Zhu). Let $A$ be a semisimple Hopf algebra and e a primitive idempotent in the character algebra $C(A)$. Then $\operatorname{dim}\left(A^{*} e\right)$ divides $\operatorname{dim}(A)$.

Theorem 3.2. Let $(A, R)$ be a factorizable Hopf algebra. If $A$ is a semisimple algebra and $V$ a simple left $A$-module, then $\operatorname{dim}(V)^{2}$ divides $\operatorname{dim}(A)$.

Proof. By assumption, $A \cong M_{d_{1}}(k) \times \cdots \times M_{d_{t}}(k)$ is isomorphic to a product of full matrix rings $M_{d_{i}}(k)$, and $Z(A) \cong k \times \cdots \times k$. Let $E_{i}$ be the primitive idempotent in $Z(A)$ corresponding to the simple module $V$. Thus $A E_{i} \cong M_{d_{i}}(k)$ has dimension $d_{i}^{2}$, and $d_{i}=\operatorname{dim}(V)$. By Theorem [2.1, $\Phi_{R}$ induces an algebra isomorphism $C(A) \rightarrow Z(A)$ (Note that here unimodularity of $A$ is very easy to see since $A$ is semisimple). Let $e_{i}$ be the primitive idempotent in $C(A)$ with $\Phi\left(e_{i}\right)=E_{i}$. 
Then by Theorem 2.1 (b), $\Phi\left(A^{*} e_{i}\right)=\Phi\left(A^{*}\right) \Phi\left(e_{i}\right)=A E_{i}$. Since $\Phi$ is bijective, $\operatorname{dim}\left(A^{*} e_{i}\right)=\operatorname{dim}\left(A E_{i}\right)=(\operatorname{dim}(V))^{2}$. Therefore $(\operatorname{dim}(V))^{2} \operatorname{divides} \operatorname{dim}(A)$ by Theorem 3.1 .

Corollary 3.3 (Etingof-Gelaki [EG]). (1) If $H$ is a semisimple Hopf algebra and $V$ a simple left $D(H)$-module, then $\operatorname{dim}(V)$ divides $\operatorname{dim}(H)$.

(2) If $(H, R)$ is a quasitriangular Hopf algebra and $H$ is semisimple, then for any simple left $H$-module, $\operatorname{dim}(V)$ divides $\operatorname{dim}(H)$.

Proof. (1) Since the characteristic of $k$ is $0, D(H)$ is semisimple by [LR. Hence by Theorem 3.2 applied to the factorizable Hopf algebra $(D(H), R),(\operatorname{dim}(V))^{2}$ divides $\operatorname{dim}(D(H))=(\operatorname{dim}(H))^{2}$. Thus $\operatorname{dim}(V)$ divides $\operatorname{dim}(H)$.

(2) follows from (1) as in [EG] since $H$ is an epimorphic image of $D(H)$.

Remark 3.4. The original proof of Corollary 3.3 (1) uses the theory of modular categories. In particular, in the first line of the proof of [EG, Lemma 1.2], the authors need a trace formula in modular categories. For a proof they refer to $\mathrm{Ki}$ who refers to Turaev's book [T]. The lucky reader may find a proof in [T, II, 3.2.2.(ii)] making use of various twistings in the graphical calculus.

However, in the case of the modular category of all finite-dimensional representations of a semisimple factorizable Hopf algebra $(A, R)$ (in [EG] $A$ is the Drinfeld double of a semisimple Hopf algebra $H$ ), this formula is in fact a special case of the class equation 3.1 in the version of Lorenz $[\mathrm{L}$.

Let $V_{i}, 0 \leq i \leq m$, with $V_{0}:=k$, be a complete set of representatives of the isomorphism classes of the simple left $A$-modules. For all $i$, let $\chi_{i}$ be the character of $V_{i}$, and let $\chi_{i^{*}}=S\left(\chi_{i}\right)$ be the character of $\left(V_{i}\right)^{*}$. By definition, the matrix $\left(s_{i j}\right)$ in [EG] is given by

$$
s_{i j}=\chi_{i}\left(\Phi\left(\chi_{j^{*}}\right)\right), 0 \leq i, j \leq m,
$$

where $\Phi=\Phi_{R}$. The trace formula needed in the proof of [EG] Lemma 1.2] then is:

$$
\text { For all } j, \sum_{i} s_{j i} s_{i j^{*}}=\operatorname{dim}(A) \text {. }
$$

To prove this formula, let $E_{i}$ in $Z(A)$ be the central primitive idempotent of $V_{i}, 0 \leq i \leq m$. For all $i$, define $e_{i}:=\Phi^{-1}\left(E_{i}\right)$, and let $\mu_{i}$ be the character of the simple $C(A)$-module $C(A) e_{i}$. Since $\Phi$ defines an algebra isomorphism between $C(A)$ and $Z(A), \pi_{i}:=\mu_{i} \Phi^{-1}$ is the character of the simple $Z(A)$-module $Z(A) E_{i}$. Note that for any $a \in Z(A)$, left multiplication with $a$ on $V_{i}$ is multiplication with $\pi_{i}(a)$; hence $\chi_{i}(a)=\pi_{i}(a) \operatorname{dim}\left(V_{i}\right)$ (for, since $V_{i}$ is simple, left multplication with $a$ on $V_{i}$ is multiplication with some scalar; since $A E_{i} \cong V_{i}^{d_{i}}, d_{i}:=\operatorname{dim}\left(V_{i}\right)$, and $Z(A) E_{i}$ is one-dimensional, this scalar is $\left.\pi_{i}(a)\right)$.

Hence for all $i, j$,

$$
s_{i j}=\chi_{i}\left(\Phi\left(\chi_{j^{*}}\right)\right)=\pi_{i}\left(\Phi\left(\chi_{j^{*}}\right)\right) \operatorname{dim}\left(V_{i}\right)=\mu_{i}\left(\chi_{j^{*}}\right) \operatorname{dim}\left(V_{i}\right),
$$

since $\Phi\left(\chi_{j^{*}}\right)$ is in the center of $A$ by Theorem 2.1 .

Moreover it is not difficult to see that for all $i, j, s_{i j}=s_{j i}$, and $s_{i j}=s_{i^{*} j^{*}}$; hence $s_{i j^{*}}=s_{j i^{*}}$ (cf. [Ki]).

Therefore one obtains

$$
\sum_{i} s_{j i} s_{i j^{*}}=\sum_{i} s_{j i} s_{j i^{*}}=\sum_{i} \mu_{j}\left(\chi_{i^{*}}\right) \mu_{j}\left(\chi_{i}\right)\left(\operatorname{dim}\left(V_{j}\right)\right)^{2} .
$$


Finally, as in the proof of Theorem 3.2, by Theorem[2.1(b), $\operatorname{dim}\left(A^{*} e_{j}\right)=\left(\operatorname{dim}\left(V_{j}\right)\right)^{2}$. Thus the trace formula is equivalent to the equality

$$
\sum_{i} \mu_{j}\left(\chi_{i^{*}}\right) \mu_{j}\left(\chi_{i}\right)=\frac{\operatorname{dim}(A)}{\operatorname{dim}\left(A^{*} e_{j}\right)}
$$

which is exactly the formula

$$
\omega(\widetilde{e})=d
$$

(for left modules) in the proof of the class equation in $\left[\mathrm{L}\right.$, where $\widetilde{e}=e_{j}=$ $\sum_{i} \mu_{j}\left(\chi_{i^{*}}\right) \chi_{i}$ and $\omega=\mu_{j}$, since here $C(A)$ is commutative.

\section{The Drinfeld double of a factorizable Hopf algebra}

If $(A, R)$ is a finite-dimensional quasitriangular Hopf algebra, define $\pi_{R}: D(A) \rightarrow$ $A$ by $\pi_{R}(f a):=f\left(R^{1}\right) R^{2} a$ for all $f \in A^{*}, a \in A$. (See 2.4 for notations concerning $D(A)$.) This map appears in [D Proof of Proposition 6.2] where it was observed that $\pi_{R}$ is a Hopf algebra homomorphism. Since $\widetilde{R}:=\left(R_{21}\right)^{-1}=S^{-1}\left(R^{2}\right) \otimes$ $R^{1}=R^{2} \otimes S\left(R^{1}\right)$ is another $R$-matrix for $A[\mathrm{~K}$, VIII.2], that is $(A, \widetilde{R})$ is again quasitriangular, there is always a second projection $\pi_{\widetilde{R}}: D(A) \rightarrow A$.

Finally, if $\varrho: A \rightarrow B$ is a homomorphism of bialgebras, then $A^{\text {co } \varrho}:=\{x \in A \mid$ $\left.x_{1} \otimes \varrho\left(x_{2}\right)=x \otimes 1\right\}$.

Lemma 4.1. Let $(A, R)$ be a finite-dimensional quasitriangular Hopf algebra. Define $\pi:=\pi_{R}, \widetilde{\pi}:=\pi_{\widetilde{R}}, \Phi:=\Phi_{R}$, and let $i: D(A)^{c o \pi} \rightarrow D(A)$ be the inclusion map. Then:

(1) $\Psi: A^{*} \rightarrow D(A)^{c o \pi}, \Psi(f):=f_{2} S\left(\pi\left(f_{1}\right)\right)$, is bijective.

(2) $S \Phi=\tilde{\pi} i \Psi: A^{*} \stackrel{\Psi}{\longrightarrow} D(A)^{c o \pi} \stackrel{i}{\longrightarrow} D(A) \stackrel{\widetilde{\pi}}{\longrightarrow} A$.

Proof. (1) The map $\Psi$ is well-defined since for all $f \in A^{*}$,

$$
\Psi(f)_{1} \otimes \pi\left(\Psi(f)_{2}\right)=f_{4} S\left(\pi\left(f_{1}\right)\right) \otimes \pi\left(f_{3} S\left(\pi\left(f_{2}\right)\right)\right)=\Psi(f) \otimes 1,
$$

since $\pi$ is a Hopf algebra homomorphism. The inverse of $\Psi$ is given by id $\otimes \varepsilon$ since for all $f \in A^{*},(\operatorname{id} \otimes \varepsilon) \Psi(f)=f$, and if $x=\sum_{i} f_{i} a_{i} \in D(A)^{c o \pi}, f_{i} \in A^{*}$ and $a_{i} \in A$ for all $i$, then $\sum_{i} f_{i 2} a_{i 1} \otimes \pi\left(f_{i 1} a_{i 2}\right)=x \otimes 1$; hence

$$
\left.x=\sum_{i} f_{i 2} a_{i 1} S \pi\left(a_{i 2}\right) S \pi\left(f_{i 1}\right)\right)=\Psi\left(\sum_{i} f_{i} \varepsilon\left(a_{i}\right)\right) .
$$

(2) For any $f \in A^{*}$,

$$
\begin{aligned}
\widetilde{\pi}(\Psi(f)) & =\widetilde{\pi}\left(f_{2} f_{1}\left(R^{1}\right) S\left(R^{2}\right)\right) \\
& =f_{2}\left(S^{-1}\left(r^{2}\right)\right) r^{1} f_{1}\left(R^{1}\right) S\left(R^{2}\right) \\
& =S\left(R^{2} S^{-1}\left(r^{1}\right) f\left(R^{1} S^{-1}\left(r^{2}\right)\right)\right. \\
& =S\left(R^{2} r^{1} f\left(R^{1} r^{2}\right)\right) \quad \text { since } S^{-1}\left(r^{1}\right) \otimes S^{-1}\left(r^{2}\right)=r^{1} \otimes r^{2} \\
& =S(\Phi(f)) .
\end{aligned}
$$

Let $H$ be a bialgebra with comultiplication $\Delta$ and augmentation $\varepsilon$. An invertible element $F \in H \otimes H$ is called a 2-cocycle for $H$ or a gauge transformation if

$$
\begin{aligned}
F_{12}(\Delta \otimes \mathrm{id})(F) & =F_{23}(\mathrm{id} \otimes \Delta)(F) \text { in } H \otimes H \otimes H, \text { and } \\
\varepsilon\left(F^{1}\right) F^{2} & =1=F^{1} \varepsilon\left(F^{2}\right) .
\end{aligned}
$$


If $F$ is a 2-cocycle for $H$, then the twisted bialgebra $H^{F}$ is defined as follows. $H^{F}=H$ as an algebra with the new comultiplication

$$
\Delta_{F}(x):=F \Delta(x) F^{-1} \text { for all } x \in H,
$$

and the old augmentation $\varepsilon$. If $H$ is a Hopf algebra, then $H^{F}$ is a Hopf algebra with antipode $S_{F}(x):=v S(x) v^{-1}$ for all $x \in H$, where $v:=F^{1} S\left(F^{2}\right), v^{-1}=$ $S\left(G^{1}\right) G^{2}, G:=F^{-1}$ (see [K, XV.3 and XV.6]).

Theorem 2.9 in $\mathrm{RS}$ describes the Drinfeld double of a factorizable Hopf algebra $A$ as a 2-cocycle twist of the usual (componentwise) tensor product bialgebra $A \otimes A$ without giving an explicit proof.

In the case when $A=D(H)$ is itself the Drinfeld double of another Hopf algebra $H$, Tsang and Zhu [TZ, Theorem 2] recently rediscovered this result. Their proof depends on the structure of $A$ as the double of $H$.

In the next theorem a stronger result is shown by using the concept of Hopf modules in the next lemma. Factorizable Hopf algebras can in fact be characterized by the property that their double is isomorphic (in a specific way) to a 2-cocycle twist of the usual tensor product bialgebra.

Lemma 4.2. Let $A, B$ and $D$ be bialgebras and $\varphi: D \rightarrow A, \psi: D \rightarrow B$ bialgebra maps. Define $\delta: D \rightarrow A \otimes B$ by $\delta(x):=\varphi\left(x_{1}\right) \otimes \psi\left(x_{2}\right)$ for all $x \in D$.

(1) If $(D, R)$ is a quasitriangular Hopf algebra, then

$$
F:=1 \otimes \psi\left(S\left(R^{1}\right)\right) \otimes \varphi\left(R^{2}\right) \otimes 1
$$

is a 2-cocycle for $A \otimes B$ with componentwise bialgebra structure, and $\delta: D \rightarrow$ $(A \otimes B)^{F}$ is a bialgebra map.

(2) Assume that $B$ is a Hopf algebra and that there is a bialgebra homomorphism $\gamma: B \rightarrow D$ with $\psi \gamma=i d_{B}$. Then $\delta: D \rightarrow A \otimes B$ is bijective if and only if the restriction of $\varphi$ defines an isomorphism between $D^{\text {co } \psi}$ and $A$.

Proof. (1) can be checked directly.

(2) It is easy to see that $\delta: D \rightarrow A \otimes B$ is a map of right $(B, B)$-Hopf modules. Here $D$ is a right $B$-module by restriction via $\gamma: B \rightarrow D$, and a right $B$-comodule by (id $\otimes \psi) \Delta ; A \otimes B$ is a right $B$-comodule by id $\otimes \Delta$, and the right $B$-module structure is given by $(a \otimes b) \cdot c:=a \varphi\left(\gamma\left(c_{1}\right)\right) \otimes b c_{2}$ for all $a \in A$ and $b, c \in B$. Hence by the theorem on Hopf modules [M] 1.9], $\delta$ is bijective if and only if $D^{c o B}=$ $D^{c o \psi} \rightarrow(A \otimes B)^{c o B} \cong A, x \mapsto \varphi(x)$, is bijective.

Theorem 4.3. Let $(A, R)$ be a finite-dimensional quasitriangular Hopf algebra. Define $F_{R}:=1 \otimes R^{2} \otimes R^{1} \otimes 1$ and $\delta_{R}: D(A) \rightarrow A \otimes A, x \mapsto \delta_{R}(x):=\widetilde{\pi}\left(x_{1}\right) \otimes$ $\pi\left(x_{2}\right)$ with $\pi:=\pi_{R}$ and $\widetilde{\pi}:=\pi_{\widetilde{R}}$. Then $F_{R}$ is a 2-cocycle for $A \otimes A$ with componentwise bialgebra structure,

$$
\delta_{R}: D(A) \rightarrow(A \otimes A)^{F_{R}}
$$

is a Hopf algebra homomorphism, and the following are equivalent:

(1) $\delta_{R}$ is bijective.

(2) $(A, R)$ is factorizable.

Proof. Let $\left(e_{i}\right)$ and $\left(f_{i}\right)$ be dual bases of $A$ and $A^{*}$. Apply Lemma 4.2 with $D:=$ $D(A)$ and $R$-matrix $\sum_{i} e_{i} \otimes f_{i}, \varphi:=\widetilde{\pi}, \psi:=\pi$, and $\gamma: A \rightarrow D(A)$ the inclusion 
map. The 2-cocycle of Lemma 4.2 (1) then is

$$
\begin{aligned}
\sum_{i} 1 \otimes \pi\left(S\left(e_{i}\right)\right) \otimes \widetilde{\pi}\left(f_{i}\right) \otimes 1 & =\sum_{i} 1 \otimes S\left(e_{i}\right) \otimes f_{i}\left(R^{2}\right) S\left(R^{1}\right) \otimes 1 \\
& =1 \otimes S\left(\sum_{i} e_{i} f_{i}\left(R^{2}\right)\right) \otimes S\left(R^{1}\right) \otimes 1 \\
& =1 \otimes S\left(R^{2}\right) \otimes S\left(R^{1}\right) \otimes 1 \\
& =F_{R} .
\end{aligned}
$$

By Lemma 4.2 (2) and Lemma 4.1, conditions (1) and (2) are both equivalent to the bijectivity of the map $D(A)^{c o \pi} \rightarrow A, x \mapsto \widetilde{\pi}(x)$.

Remark 4.4. Radford [R94, Proposition 3] showed that a factorizable Hopf algebra is unimodular. Another proof of this result follows from the isomorphism $D(A) \cong$ $(A \otimes A)^{F_{R}}$ in Theorem 4.3 and the unimodularity of the double [R93, Theorem4].

Let $\Lambda$ be a left integral in $A$. Then $\Lambda \otimes \Lambda$ is a left integral in $(A \otimes A)^{F_{R}}$. Since $D(A)$ is unimodular, $\Lambda \otimes \Lambda$ is a right integral. Hence $\Lambda$ is a right integral in $A$.

\section{REFERENCES}

[D] V. G. Drinfeld, On almost cocommutative Hopf algebras, Leningrad Math. J. 1 (1990), 321-342.

[EG] P. Etingof and S. Gelaki, Some properties of finite-dimensional semisimple Hopf algebras, Mathematical Research Letters 5 (1998), 191-197. MR 99e:16050

[K] C. Kassel, Quantum Groups, Springer, GTM 155, New York, 1995. MR 96e:17041

[Ki] A. A. Kirillov Jr., On an inner product in modular categories, J. Amer. Math. Soc. 9 (1996), 1135-1169. MR 97f:18007

[LR] R. G. Larson and D. E. Radford, Finite-dimensional cosemisimple Hopf algebras in characteristic 0 are semisimple, J. Alg. 117 (1988), 267-289. MR 89k:16016

[L] M. Lorenz, On the class equation for Hopf algebras, Proc. Amer. Math. Soc. 126 (1998), 2841-2844. MR 99a:16033

[M] S. Montgomery, Hopf algebras and their actions on rings, CBMS Lecture Notes 82, Amer. Math. Soc., 1993. MR 94i:16019

[OS] U. Oberst, H.-J. Schneider, Über Untergruppen endlicher algebraischer Gruppen, manuscripta math. 8 (1973), 217-241. MR 50:339

[R93] D. E. Radford, Minimal quasitriangular Hopf algebras, J. Algebra 157 (1993), 285-315. MR 94c:16052

[R94] D. E. Radford, On Kauffman's knot invariants arising from finite-dimensional Hopf algebras, in: Advances in Hopf Algebras, Lecture Notes in Pure and Applied mathematics, Dekker, New York, 1994, 205-266. MR 96g:57013

[RS] N. Y. Reshetikhin, M. A. Semenov-Tian-Shansky, Quantum R-matrices and factorization problems, Journal Geometry Physics 5 (1988), 533-550. MR 92g:17019

[TZ] Y. Tsang, Y. Zhu, On the Drinfeld double of a Hopf algebra, preprint, 1998.

$[\mathrm{T}]$ V. Turaev, Quantum invariants of knots and 3-manifolds, de Gruyter Studies in Mathematics 18, Walter de Gruyter \& Co., Berlin, 1994.

Mathematisches Institut, Universität MÜnchen, Theresienstrasse $39, \quad$ D-80333 MünChen, Germany

E-mail address: hanssch@rz.mathematik.uni-muenchen.de 